\title{
Maturation of mouse fetal germ cells in vitro
}

\section{Even immature oocytes can eventually be fertilized after some skilful manipulation.}

N uclear reprogramming is essential during gametogenesis for the production of totipotent zygotes. Here we show that premeiotic female germ cells derived from mouse fetuses as early as 12.5 days post coitum are able to complete meiosis and genomic imprinting in vitro and that these matured oocytes are highly competent in supporting development to full term after nuclear transfer and in vitro fertilization. To our knowledge, this is the first time that complete oogenesis has been successfully accomplished in vitro.

Although the ovaries of mammals contain thousands or millions of immature oocytes, few of these ever mature to the point at which reproduction in vivo is possible. Ovarian oocytes therefore constitute a large and potentially valuable resource for clinical and zoological application. However, although mature oocytes have been produced in vitro by culturing immature oocytes $^{1-3}$, oogenesis was never complete, and attempts to produce even non-growing oocytes at the diplotene stage of the first meiosis from ovaries derived from newborn mice have met with limited success (only $0.016 \%$; ref. 2).

We have shown that this poor ability of oocytes to mature in culture is due to their incompetent cytoplasm ${ }^{4}$, a problem that can be overcome by transferring their nuclei into enucleated, fully grown oocytes. Although live pups can eventually be produced from oocytes reconstituted in this way, this is not possible if nuclei from small, immature oocytes are used for reconstitution, probably because of defects in their meiotic chromosomal configuration and/or genomic imprinting ${ }^{5-7}$.

We cultured premeiotic female germ cells in vitro in an attempt to complete this essential nuclear reprogramming (see supplementary information for details). Ovaries from mouse fetuses at 12.5 days post coitum (d.p.c.), with the mesonephroi attached, were cultured for 7 days, followed by removal of the mesonephroi and a further 10 days in culture (Fig. 1). These cultured ovaries contained many secondary follicles, which we isolated and cultured for 11 days; at the end of the 28-day culture period, some follicles showed antrum formation and the oocytes had increased in diameter (63.9 $\mu \mathrm{m}, n=127$; Fig. 1$)$.

We estimated the extent of genomic imprinting in these oocytes by analysing DNA methylation in the imprinted gene $\operatorname{Igf} 2 r$ (ref. 8). The methylation pattern at each stage of culture was consistent with that in oocytes taken from mice at the
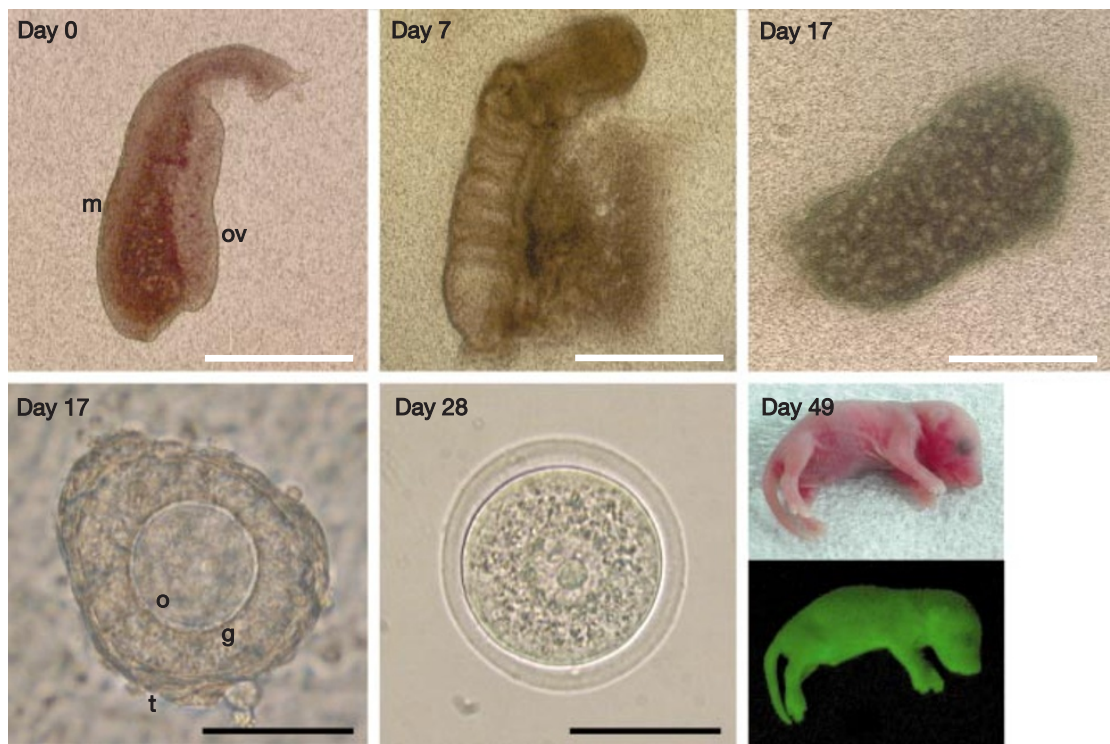

Figure 1 Development of premeiotic female germ cells in vitro. Day 0, ovary (ov) from a green-fluorescent-protein (GFP)-labelled B6CBF1 $\left(\right.$ GFP-C57BL/6J $\times$ CBA) transgenic mouse fetus at 12.5 d.p.c. ${ }^{11}$, with attached mesonephroi $(\mathrm{m})$. Ovaries were cultured in Waymouth medium supplemented with $10 \%$ fetal bovine serum (FBS) on a Costar Transwell membrane ${ }^{2}$. At day 7 the mesonephroi was removed and at day 17 a secondary follicle, consisting of theca cells (t) and 2-3 layers of granulosa cells (g) around the 0ocyte (0), was isolated from the ovary and cultured in MEM- $\alpha$ medium supplemented with $5 \% \mathrm{FBS}, 0.1 \mathrm{IU} \mathrm{ml}^{-1}$ follicle-stimulating hormone, $5 \mu \mathrm{g} \mathrm{ml}^{-1}$ insulin, $5 \mu \mathrm{g} \mathrm{ml}^{-1}$ transferrin and $5 \mathrm{ng} \mathrm{ml}^{-1}$ selenium on a Costar Transwell-COL membrane ${ }^{2,3}$. Day 28, oocyte isolated from cultured follicle for nuclear transfer. After nuclear transfer and in vitro fertilization, the resulting zygotes (bottom right. day 49) were cultured in M16 medium. All cultures were incubated at $37^{\circ} \mathrm{C}$ in an atmosphere of $5 \% \mathrm{CO}_{2}$ in air. Scale bars: white, $500 \mu \mathrm{m}$; black, $50 \mu \mathrm{m}$.

same stage (see supplementary information), indicating that normal imprinting can be established in vitro.

We also investigated whether nuclear reprogramming could occur in these oocytes by monitoring their development (see supplementary information). Because oocytes isolated from cultured follicles were unable to resume meiosis $(0 / 38,0 \%)$, we transferred the nuclei into enucleated, fully grown oocytes from adult mice. These reconstituted oocytes were able to resume meiosis and mature into metaphase in the second meiosis (M II, 101/108, 94\%) and had a normal karyotype $(n=20$, $11 / 11,100 \%)$, indicating that chromosomal maturation for meiosis can be completed in vitro. As expected ${ }^{4}$, oocytes reconstituted with intact female germ cells from fetuses at 12.5 d.p.c. became aneuploid (32/32, $100 \%$ ) owing to abnormal chromosomal segregation.

As oocytes reconstituted by a single nuclear transfer did not develop efficiently into blastocysts $(14 / 34,41 \%)$, we used serial nuclear transfer (see supplementary information for details). The rate of in vitro fertilization of these reconstituted oocytes was normal $(72 / 81,89 \%)$ and they developed efficiently into blastocysts $(64 / 72,89 \%)$.
After embryo transfer to a surrogate mother, 16 living pups were obtained from 7 mothers at 19.0 d.p.c. by caesarean section (16/64, 25\%). No obvious abnormality was seen in any of the pups (in vitro pup weight compared with that in vivo: $1.46 \mathrm{~g}$ versus $1.34 \mathrm{~g}, P>0.1$ ) or placentae (in vitro weight compared with that in vivo: $133 \mathrm{mg}$ versus $126 \mathrm{mg}, P>0.5$ ), as expected from normal imprinted methylation of Igf2r, Snrpn and Peg1 (see supplementary information) $)^{9,10}$. These animals were fertile after puberty.

We have shown that the most primitive murine fetal oocytes can differentiate into competent oocytes with high efficiency. As well as offering an opportunity to analyse the mechanisms behind nuclear reprogramming in vitro, our system might eventually help women undergoing chemotherapy or radiotherapy to become mothers afterwards, by prior removal of an ovary.

\section{Yayoi Obata*, Tomohiro Kono ${ }^{\star}$,}

Izuho Hatada ${ }^{\star}$

${ }^{\star}$ Gene Research Center, Gunma University,

3-39-22 Showa-machi, Maebashi,

Gunma 371-8511, Japan

e-mail:ihatada@showa.gunma-u.ac.jp $\dagger$ Department of Bioscience, Tokyo University of

Agriculture, 1-1-1 Sakuragaoka, Setagaya-ku,

Tokyo 156-8502, Japan 
1. Buehr, M. \& McLaren, A. Gamete Res. 11, 271-281 (1985).

2. Eppig, J. J. \& O'Brien, M. J. Biol. Reprod. 54, 197-207 (1996).

3. Cortvrindt, R., Smitz, J. \& Van Steirteghem, A. C. Hum. Reprod. 11, 2656-2666 (1996).

4. Bao, S., Obata, Y., Carroll, J., Domeki, I. \& Kono, T. Biol. Reprod. 62, 616-621 (2000)

5. Kono, T., Obata, Y., Yoshimzu, T., Nakahara, T. \& Carroll, J. Nature Genet. 13, 91-94 (1996)

6. Obata, Y. et al. Development 125, 1553-1560 (1998).

7. Obata, Y. \& Kono, T. J. Biol. Chem. 277, 5285-5289 (2002).

\section{Gene silencing}

\section{Trans-histone regulatory pathway in chromatin}

- he fundamental unit of eukaryotic chromatin, the nucleosome, consists of genomic DNA wrapped around the conserved histone proteins $\mathrm{H} 3, \mathrm{H} 2 \mathrm{~B}, \mathrm{H} 2 \mathrm{~A}$ and $\mathrm{H} 4$, all of which are variously modified at their amino- and carboxy-terminal tails to influence the dynamics of chromatin structure and function ${ }^{1,2}$ - for example,

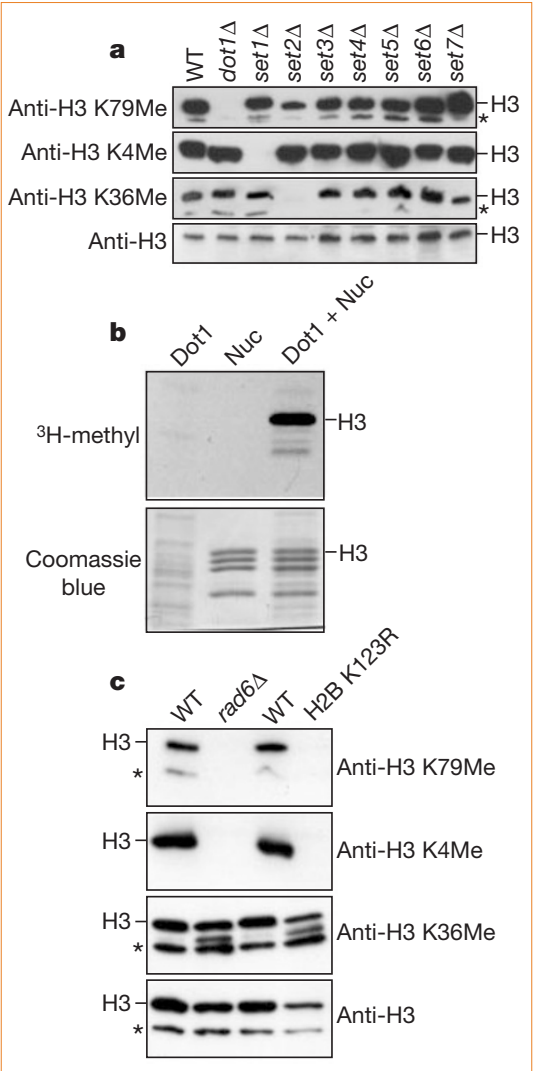

Figure 1 Regulation of Lys-79 methylation on histone $\mathrm{H} 3$. a, Western blot of nuclear extracts isolated from mutant yeast strains carrying the indicated deletions and probed with antibodies against the methylated $\mathrm{H} 3$ lysine residues K79Me, K4Me or K36Me. b, Polyacrylamide-gel electrophoresis analysis of recombinant Dot1 for histone methyltransferase activity on nucleosomal substrates (Nuc) in vitro, as revealed by autoradiography (top) and Coomassie-blue staining of histones (bottom). c, Western blot of nuclear extracts from wild-type yeast and rad6-deleted or H2B K123R mutant strains probed with the histone antibodies indicated. Asterisks, an $\mathrm{H} 3$ proteolysis product that removes the methylation site at Lys 4 but not at Lys 36 or Lys 79 .
Wutz, A et al Nature 389, 745-749 (1997).

9. Bourc'his, D., Xu, G. L., Lin, C. S., Bollman, B. \& Bestor, T. H. Science 294, 2536-2539 (2001).

10.Lucifero, D., Mertineit, C., Clarke, H. J., Bestor, T. H. \& Trasler, J. M. Genomics 79, 530-538 (2002).

11. Okabe, M., Ikawa, M., Kominami, K., Nakanishi, T. \& Nishimune, Y. FEBS Lett. 407, 313-319 (1997).

Supplementary information accompanies this communication on Nature's website.

Competing financial interests: declared none.

conjugation of histone $\mathrm{H} 2 \mathrm{~B}$ with ubiquitin controls the outcome of methylation at a specific lysine residue (Lys 4) on histone $\mathrm{H} 3$, which regulates gene silencing in the yeast Saccharomyces cerevisiae ${ }^{3}$. Here we show that ubiquitination of $\mathrm{H} 2 \mathrm{~B}$ is also necessary for the methylation of Lys 79 in $\mathrm{H} 3$, the only modification known to occur away from the histone tails, but that not all methylated lysines in $\mathrm{H} 3$ are regulated by this 'trans-histone' pathway because the methylation of Lys 36 in H3 is unaffected. Given that gene silencing is regulated by the methylation of Lys 4 and Lys 79 in histone $\mathrm{H} 3$, we suggest that $\mathrm{H} 2 \mathrm{~B}$ ubiquitination acts as a master switch that controls the site-selective histone methylation patterns responsible for this silencing.

Lysine residues subject to methylation in yeast histone $\mathrm{H} 3$ are Lys 4 and Lys 36 near the amino terminus, and Lys 79 (refs 4-6, and data not shown), a modification site that is unique in that it is located away from the $\mathrm{H} 3$ tails in the first loop of the histonefold domain. To identify what mediates methylation of Lys 79, we used an antibody raised against this methylated site (anti-H3 K79Me; Upstate Biotechnology) to screen nuclear extracts isolated from yeast strains containing deletions of known and putative histone methyltransferase enzymes.

We identified Dot1, a factor involved in gene silencing ${ }^{4,5}$, as a gene product that is essential for methylation of Lys 79 (Fig. 1a), in agreement with earlier findings ${ }^{4-6}$. This was unexpected, as Dot1 lacks the 'SET' domain, which until now was thought to be the only domain responsible for methylating histone lysine residues. No other protein containing the SET domain was found to mediate Lys-79 methylation (Fig. 1a). To confirm that Dotl is the enzyme responsible, we showed that expression of DOT1 in a dot1-deleted strain restores Lys-79 methylation in $\mathrm{H} 3$ (see supplementary information) and that recombinant Dotl contained methyltransferase activity towards nucleosomal H3 (Fig. 1b). Dot1, which resembles the arginine methyltransferase family in sequence and structure ${ }^{7}$, therefore represents a new class of lysine-specific histone methyltransferase enzymes.

Ubiquitination of histone $\mathrm{H} 2 \mathrm{~B}$ is mediated by the enzyme Rad6, also known as ubiquitin-conjugating enzyme Ubc2 (ref. 8). We tested whether Rad6 ubiquitination of $\mathrm{H} 2 \mathrm{~B}$ could influence methylation at $\mathrm{H} 3$ sites apart from Lys 4 (ref. 3). Surprisingly, we found that there was a loss of Lys-79 methylation, but not of Lys-36 methylation, in strains deleted for RAD6 or mutated at the H2B-ubiquitination site (K123R; Fig. 1c). We verified that Dot1 is expressed in these mutant strains by using reverse transcription followed by polymerase chain reaction to detect the presence of its messenger RNA (see supplementary information). We conclude that Rad6 ubiquitination of $\mathrm{H} 2 \mathrm{~B}$ at Lys 123 specifically regulates the methylation of both Lys 4 and Lys 79 of H3 in a 'trans-histone' pathway.

We found that deletion of DOT1, SET1 (refs 9, 10) or SET2 (ref. 11) results in the specific loss of their respective modifications (Fig. 1a), indicating that the absence of these individual modifications does not affect the others. Given that the regulation of Lys-4 methylation by H2B ubiquitination is unidirectional ${ }^{3}$ and that Lys- 4 methylation still occurs in the dot1-deleted (Fig. 1a) and Lys-79 mutant strains (see supplementary information), we conclude that the regulation of Lys-79 methylation by H2B ubiquitination is also unidirectional.

Our findings indicate that methylation of histone $\mathrm{H} 3$ at Lys 4 and Lys 79, but not at Lys 36 , is regulated by the ubiquitination of histone H2B. As Lys 4 and Lys 79 methylation both mediate gene silencing, we propose that $\mathrm{H} 2 \mathrm{~B}$ ubiquitination acts as a master switch of gene silencing through a trans-histone pathway that leads to the appropriate patterns of histone methylation. Given that some lysine residues in $\mathrm{H} 3$ are affected by this pathway, but not others, our results lend support to the 'histone code' hypothesis ${ }^{2}$.

Scott D. Briggs ${ }^{\star}$, Tiaojiang Xiao $\dagger$, Zu-Wen Sun ${ }^{\star}$, Jennifer A. Caldwell Jeffrey Shabanowitz $\ddagger$, Donald F. Hunt $\ddagger$, C. David Allis ${ }^{\star}$, Brian D. Strahl $\dagger$

*Department of Biochemistry and Molecular Genetics, University of Virginia Health System, Charlottesville, Virginia 22908, USA $\dagger$ Department of Biochemistry and Biophysics, University of North Carolina at Chapel Hill, Chapel Hill, North Carolina 27599, USA e-mail:brian_strahl@med.unc.edu $\$$ Departments of Chemistry and Pathology,

University of Virginia, Charlottesville, Virginia 22908, USA

\footnotetext{
1. Kornberg, R. D. \& Lorch, Y. Cell 98, 285-294 (1999).

2. Strahl, B. D. \& Allis, C. D. Nature 403, 41-45 (2000).

3. Sun, Z. W. \& Allis, C. D. Nature 418, 104-108 (2002).

4. van Leeuwen, F., Gafken, P. R. \& Gottschling, D. E. Cell 109, 745-756 (2002).

5. Ng, H. H. et al. Genes Dev. 16, 1518-1527 (2002).

6. Feng, Q. et al. Curr. Biol. 12, 1052-1058 (2002).

7. Dlakic, M. Trends. Biochem. Sci. 26, 405-407 (2001).

8. Robzyk, K., Recht, J. \& Osley, M. A. Science 287, 501-504 (2000).

9. Briggs, S. D. et al. Genes Dev. 15, 3286-3295 (2001).

10. Bryk, M. et al. Curr. Biol. 12, 165-170 (2002).

11. Strahl, B. D. et al. Mol. Cell Biol. 22, 1298-1306 (2002).

Supplementary information accompanies this communication on Nature's website.

Competing financial interests: declared none.

Published online 14 July 2002; doi:10.1038/nature00970
} 